\title{
Natural Fracture Characterization Using Passive Seismic Illumination
}

\author{
Kurt T. Nihei \\ Earth Sciences Division
}

January 2003

This work was supported by the Gas Research Institute under contact 5096-260-3775. 


\begin{abstract}
The presence of natural fractures in reservoir rock can significantly enhance gas production, especially in tight gas formations. Any general knowledge of the existence, location, orientation, spatial density, and connectivity of natural fractures, as well as general reservoir structure, that can be obtained prior to active seismic acquisition and drilling can be exploited to identify key areas for subsequent higher resolution active seismic imaging. Current practices for estimating fracture properties before the acquisition of surface seismic data are usually based on the assumed geology and tectonics of the region, and empirical or fracture mechanics-based relationships between stratigraphic curvature and fracturing.
\end{abstract}

The objective of this research is to investigate the potential of multicomponent surface sensor arrays, and passive seismic sources in the form of local earthquakes to identify and characterize potential fractured gas reservoirs located near seismically active regions. To assess the feasibility of passive seismic fracture detection and characterization, we have developed numerical codes for modeling elastic wave propagation in reservoir structures containing multiple, finite-length fractures. This article describes our efforts to determine the conditions for favorable excitation of fracture converted waves, and to develop an imaging method that can be used to locate and characterize fractures using multicomponent, passive seismic data recorded on a surface array. 


\section{Contents}

1. Motivation and Plan of Investigation

2. Objectives

3. Approach

3.1 Review of Passive Seismic Methods for Imaging the Subsurface

3.2 Characteristics of a Vertically Fractured Gas Reservoir

3.3 Discrete Fracture Modeling

3.4 Seismic Visibility of Discrete Fractures

3.5 Numerical Results for a Vertically Fractured Gas Reservoir with Passive Seismic Illumination

3.6 Reverse Time Fracture Imaging with Passive P-S Converted Waves

4. Discussion and Conclusions

5. Recommendations

References 


\section{Motivation and Plan of Investigation}

The presence of natural fractures in reservoir rock can significantly enhance gas production, especially in tight gas formations. Any general knowledge of the existence, location, orientation, spatial density, and connectivity of natural fractures, as well as general reservoir structure, that can be obtained prior to active seismic acquisition and drilling can be exploited to identify key areas for subsequent higher resolution active seismic imaging. Current practices for estimating fracture properties before the acquisition of surface seismic data are usually based on the assumed geology and tectonics of the region, and empirical or fracture mechanics-based relationships between stratigraphic curvature and fracturing (Price and Cosgrove, 1990). These mechanicsbased methods can be important tools for providing general regional patterns and locations of anticipated fracturing, provided that information about the regional stratigraphy, rock properties, faults, and tectonic history is available. However, it is clear that the success of mechanics-based methods requires a fairly detailed understanding of the fracture mechanics theory and rock properties specific to the strata of the specific location being investigated, both of which can be complex. With this in mind, it is not clear what degree of confidence and what level of spatial resolution can be assigned to fracture maps produced by these methods.

Direct methods of fracture detection and characterization, such as 3-D surface seismic imaging, typically require large capital investments. The basic question posed by the Gas Research Institute is whether less expensive fracture detection and characterization methods can be developed for the purpose of prospecting for potential fractured gas reservoirs. In this study, we examine a potential method for fractured reservoir prospecting using passive seismic waves from local earthquakes. This study is both fundamental and applied in nature. We start by developing numerical modeling tools for seismic wave propagation in fractured rock leveraging off past research largely supported by the DOE Basic Energy Sciences, and then use these codes to determine the seismic signatures of vertically fractured gas reservoirs. The results of the modeling exercise motivated the development of a fracture imaging algorithm, based on the reverse time migration concept, that can be applied to multicomponent seismic array data from passive seismic sources (i.e., local earthquakes located less than $100 \mathrm{~km}$ away). Examples of the fracture imaging approach are given for a passive source that, because of the strong velocity gradient in the upper ten kilometers of the Earth, is assumed to be a vertically incident plane wave.

\section{Objectives}

The focus of this project was to develop new methods for fractured gas reservoir characterization using multicomponent surface sensor arrays and passive seismic sources. The detailed objectives of this research on fractured gas reservoir characterization are as follows: (1) to develop numerical modeling capabilities for vertically-fractured gas reservoirs and passive sources, (2) to quantify the seismic characteristics of verticallyfractured gas reservoirs for passive seismic sources (local earthquakes), and (3) to investigate the potential of surface sensor arrays, and multicomponent seismic data from 
local earthquakes to image discrete fractures in gas reservoirs.

\section{Approach}

The approach taken in this study was to use the results of prior fundamental research on elastic wave propagation in fractured rock to develop forward modeling capabilities for passive seismic waves in fractured gas reservoirs. The modeling studies were used to explore for potential diagnostic seismic signatures of a fractured gas reservoir illuminated by passive seismic waves. These results stimulated the development of a fracture imaging scheme that utilizes P-S converted waves that are recorded by a multicomponent surface sensor array. The background and details of these three efforts are described in detail the following subsections.

\subsection{Review of Passive Seismic Methods for Imaging the Subsurface}

The terms active and passive refer to the source of seismic energy used to characterize the subsurface. Active seismic methods rely on man-made sources such as vibrators, air guns, and explosives, and typically generate seismic waves with frequencies in the 20$500 \mathrm{~Hz}$ range. Over the last decade, active seismic methods have been increasingly used to characterize fracture orientation and density (see Tsvankin and Lynn, 1999 for a description of recent work). These methods are often based on relationships between theoretical models for wave propagation in an anisotropic layer and some form of processed or unprocessed surface seismic reflection data, such as normal moveout curves, shear wave splitting, and P-wave amplitude versus source-receiver azimuth and offset (AVA and AVO).

Surface seismic reflection imaging employing active sources such as explosives and vibrators is currently the method of choice for fractured reservoir detection and characterization to determine fracture orientation and density (e.g., for reviews, see Tsvankin and Lynn, 1999, and MacBeth and Lynn, 2000). Surface seismic methods for fractured reservoir characterization typically assume that the fracture spacing is small relative to the seismic wavelength. When this assumption is valid and the fractures are aligned in sets, it is possible to utilize effective medium theories to compute the (zero frequency) equivalent anisotropic properties of the fractured rock mass. When these properties are used in plane wave analysis, ray theory, and numerical wave propagation simulations, diagnostic signatures of the fracture orientation and concentration result (e.g., azimuthally-varying normal moveout, split shear waves, direction-dependent amplitude versus azimuth). Larger discontinuities such as vertical and dipping thrust and normal faults can be imaged on the processed section by analyzing the spatial coherency of the reflections from the fault-offset layers (Marfurt et al., 1998).

Passive seismic methods can be broadly divided into two classes: (1) those utilizing local, regional, and teleseismic earthquakes, and (2) those utilizing local microseismicity produced by rock fracturing during the processes of reservoir production and fluidinjection. The latter sources of seismic waves are very useful for monitoring productionrelated activities in a producing reservoir, but, unfortunately, are not useful as a means of prospecting for fractured reservoirs. In the former category, the low frequency-content of teleseismic events results in seismic waves with long wavelengths $(>2 \mathrm{~km})$ that are better 
suited for studying large-scale earth structure rather than reservoir scale structure. Local earthquakes are those events located less than approximately $100 \mathrm{~km}$ away. Even for relatively small magnitudes $\left(\mathrm{M}_{\mathrm{L}}<2.0\right)$, these events can produce observable signals at the surface with frequencies as high as $50 \mathrm{~Hz}$ (Archuleta et al., 1992). It is possible to observe much higher frequencies by avoiding the effects of the highly attenuating near surface layers with downhole receivers (Leary and Abercrombie, 1994).

While there have been a number of studies utilizing local seismicity to investigate lithosphere structure at depths of several hundred kilometers (e.g., Troitskiy et al., 1981), we are aware of only one other study (Cole, 1995) that attempted to determine shallow structures at depths of less than several kilometers. We are not aware of any studies that have attempted to use local seismicity to identify natural fractures in the subsurface.

\subsection{Characteristics of a Vertically Fractured Gas Reservoir}

Natural fractures in stratified sedimentary rocks often appear as vertical planes of compliance (Price and Cosgrove, 1990) with spacing to bed thickness ratios from less than 0.1 to greater than 10 (Bai and Pollard, 2000). Past research on the seismic properties of fractured rock has demonstrated that a gas reservoir comprised of single or multiple sets of aligned fractures can be represented by an anisotropic medium if the wavelength is large relative to the fracture spacing and the fracture equivalent background thickness (a measure of the fracture compliance relative to the host medium compliance, Haugen and Schoenberg, 2000). Using the equivalent medium representation of a fractured rock mass, a variety of diagnostic seismic wave phenomena have been predicted that can be quantified using surface seismic reflection methods such as rotational analysis of converted waves, azimuthal AVO analysis, and NMO ellipticity (Pérez et al., 1999; Tsvankin and Lynn, 1999).

If the seismic wavelength is on the order or smaller than the fracture spacing, fractures behave as localized impedance contrasts that can produce a variety of frequency and fracture compliance sensitive waves, including converted waves, fracture interface waves (coupled Rayleigh waves; Pyrak-Nolte et al., 1992; Gu et al., 1996a), and fracture channel waves (Nihei et al., 1999; Nakagawa, 1998). In this study, we focus our efforts on investigation of the effects of discrete fractures on seismic waves generated by local seismicity (i.e., passive seismic illumination). As illustrated in Figure 1, discrete fracture detection targets a subset of the fracture population that are the most compliant, and that have a spacing comparable or larger than the seismic wavelength (e.g., fracture set $\mathrm{s}_{2}$ ). Because it is likely that the larger, compliant fractures may act as conduits for enhanced production, as well as barriers that result in reservoir compartmentalization, and because the short, closely spaced fractures will be below the resolution limits of typical passive and active seismic waves that can be reliably recorded by surface sensors $(\sim 10-1000 \mathrm{~m}$ for 1-100 Hz seismic waves), we will focus our efforts here on detection of the larger, widely spaced fractures (height $100 \mathrm{~m}$, spacing $\geq 100 \mathrm{~m}$ ). 


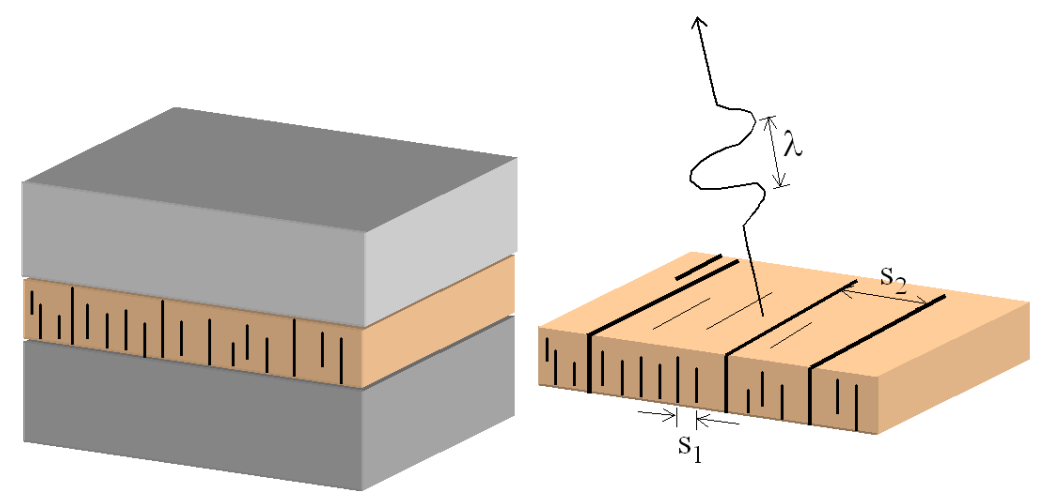

Figure 1. Conceptual model of a fractured gas reservoir containing a set of closelyspaced verical fractures with average spacing $\mathrm{s}_{1}$, and a set of larger, compliant, throughgoing fractures with average spacing $\mathrm{s}_{2}$.

\subsection{Discrete Fracture Modeling}

The numerical modeling of seismic waves in a rock mass with discrete, finite-length fractures requires an efficient and accurate numerical approach that is capable of modeling many fractures and realistic strata. Boundary element approaches probably offer the highest accuracy and flexibility of available numerical methods for modeling discrete, finite-length fractures. Fractures can be modeled explicitly as displacementjump boundary conditions (Equation 2) with spatially varying fracture compliances (Gu et al., 1996a). Additionally, special crack tip boundary elements have been developed to preserve the singular nature of the stress field at the tips of the fracture (e.g., Zhang and Achenbach, 1988). Boundary element methods require the formation of a large system matrix that is neither sparse nor symmetric. This implicit system of equations can be challenging to solve by standard numerical solvers such as LU decomposition as the number of unknowns (i.e., layers and fractures) are increased to accommodate sedimentary basin structure and scale.

A recent paper by Coates and Schoenberg (1995) has demonstrated that it is possible to accurately model discrete fractures in an explicit finite difference code. This explicit approach is fast computationally and is capable of modeling complex strata. In the following, we describe the Coates-Schoenberg approach and discuss our efforts to use this approach to model seismic wave propagation in a medium containing finite-length fractures.

\section{Coates-Schoenberg approach for discrete fracture modeling}

Discrete fracture modeling is performed using an explicit, anisotropic finite difference code based on the staggered grid method with $4^{\text {th }}$ order accuracy in space and $2^{\text {nd }}$ order accuracy in time (see Graves (1996) for a description of the staggered grid finite difference method). Using an anisotropic elastic wave propagation code, individual fractures can be represented as compliant zones of a single cell thickness (Figure 2) and with properties described by their anisotropic elastic constants (six in 2-D), as presented by Coates and Schoenberg (1995). The process for obtaining the anisotropic elastic 
constants for the finite difference cells containing the fracture and a small volume of host rock is based on a calculation of the average strain of each cell (Schoenberg and Sayers, 1995)

$$
\varepsilon_{i j}^{c e l l}=s_{i j k l}^{b} \sigma_{k l}+\frac{1}{2 L}\left(\left[u_{i}\right] n_{j}+\left[u_{j}\right] n_{i}\right),
$$

where $s_{i j k l}^{b}$ is the compliance of the host rock encompassing the fracture, $\sigma_{k l}$ is the stress, $\left[u_{i}\right]$ is the displacement jump across the fracture (i.e., the displacement-discontinuity or crack opening displacement), $L$ is the length of the cell, and $n_{i}$ is the fracture normal vector.
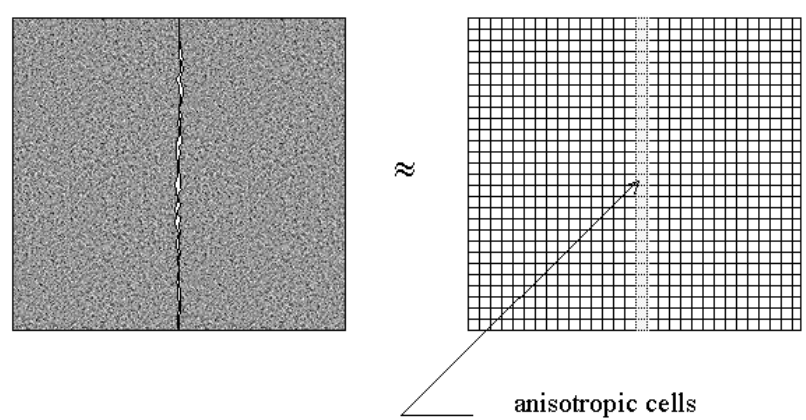

Figure 2. Representation of a discrete, vertical fracture in a finite difference model as a single column of anisotropic cells. The elastic constants for these cells are computed using the Coates-Schoenberg approach described in the text.

Assuming that the fracture deforms in an elastic fashion, the applied traction $\sigma_{j k} n_{k}$ and the resulting displacement jump $\left[u_{i}\right]$ are linearly-related through a constant $Z_{i j}$,

$$
\left[u_{i}\right]=Z_{i j} \sigma_{j k} n_{k}
$$

Here, $Z_{i j}$ is the fracture compliance, and its inverse is the fracture stiffness $K_{i j}$. Equation 1 becomes

$$
\begin{aligned}
\varepsilon_{i j}^{\text {cell }} & =s_{i j k l}^{b} \sigma_{k l} \\
& +\frac{1}{4 L}\left(Z_{i k} n_{l} n_{j}+Z_{j k} n_{l} n_{i}+Z_{i l} n_{k} n_{j}+Z_{j l} n_{k} n_{i}\right) \sigma_{k l} \\
& \equiv S_{i j k l}^{\text {cell }} \sigma_{k l} .
\end{aligned}
$$

Here, $s_{i j k l}^{\text {cell }}$ are the anisotropic compliances for the cell containing the fracture.

The compliance matrix given in Equation 3 can be inverted to give the anisotropic elastic constants of the cell containing the host+fracture. For a vertical fracture with its normal in the 1-direction, there are 4 independent anisotropic elastic constants for a 2-D model in the 1-3 plane 


$$
c_{I J}^{c e l l}=\left[\begin{array}{ccc}
(\lambda+2 \mu)\left(1-\delta_{N}\right) & \lambda\left(1-\delta_{N}\right) & 0 \\
\lambda\left(1-\delta_{N}\right) & (\lambda+2 \mu)\left(1-r^{2} \delta_{N}\right) & 0 \\
0 & 0 & \mu\left(1-\delta_{T}\right)
\end{array}\right],
$$

where

$$
\begin{aligned}
r & =v /(1-v), \\
\delta_{N} & =Z_{N}(\lambda+2 \mu) /\left[L+Z_{N}(\lambda+2 \mu)\right], \\
\delta_{T} & =Z_{T} \mu /\left(L+Z_{T} \mu\right),
\end{aligned}
$$

and $v, \lambda$, and $\mu$ are the Poisson's ratio, Lamé constant and shear modulus of the host rock, $Z_{N}$ and $Z_{T}$ are the normal and shear fracture compliances, and $L$ is the length of the finite difference cell. Equation 4 describes the vertical fracture as a transversely isotropic material with a horizontal axis of symmetry (HTI material). Because individual fractures are described by their anisotropic elastic constants, multiple discrete fractures with any specified normal and shear fracture compliances can be easily modeled. Fractures oriented at an angle to the finite difference grid can also be modeled by applying a rotation transformation to Equation 4, as described by Coates and Schoenberg (1995).

\section{Modeling finite-length fractures}

Finite-length fractures are currently being modeled using the Coates-Schoenberg finite difference approach outlined in the previous section by simply truncating the fracture without any special considerations for the fracture tips. The physical meaning of this type of fracture tip model within the context of elliptical versus cusp-shaped crack opening displacements (i.e., elastic crack tip model versus Dugdale-Barenblatt elastoplastic crack tip model; Kanninen and Popelar, 1985) is the subject of future research and beyond the scope of this project. This issue may be somewhat academic, however, because the numerical simulations shown here indicate that the fracture tip diffracted waves are not the dominant signature of the fracture for waves recorded in the far-field.

The results for a finite-length fracture generated by the finite difference approach have been compared with a 2-D elastic boundary element code (multi-region formulation) in which the fracture is modeled using the displacement jump boundary condition described by Equation 2. Qualitative comparisons show good agreement for the body wave phases with small differences in the amplitudes of the various crack-tip diffracted phases (Figure $3)$. 


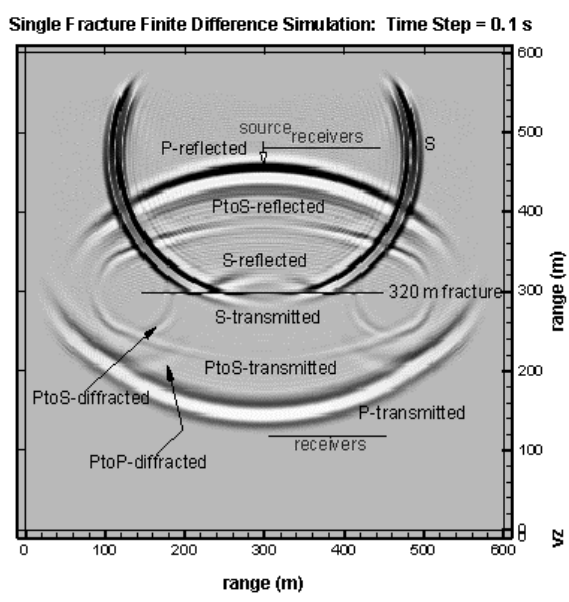

(a)

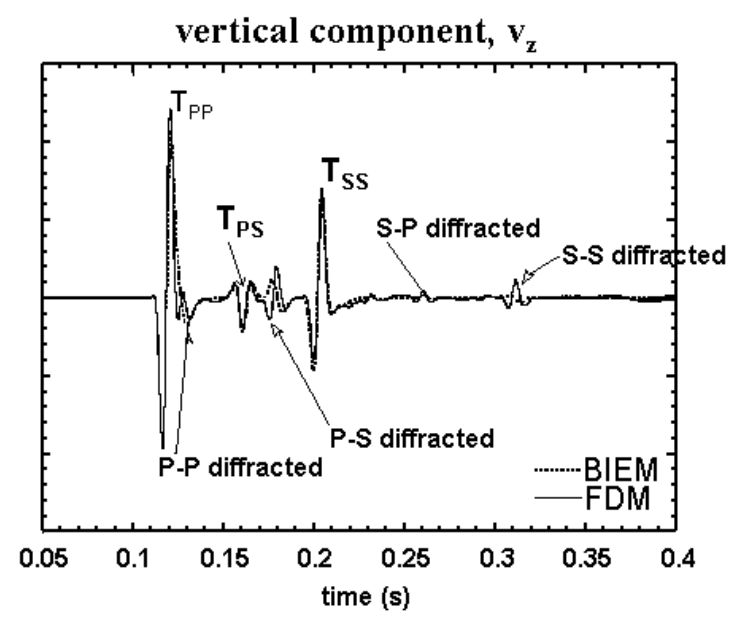

(b)

Figure 3. Comparison between the finite difference method and boundary element method for modeling 2-D elastic wave propagation across a single fracture: (a) fracture model with snapshot of the vertical particle velocity superimposed, and (b) vertical component of the particle velocity computed using the two numerical methods. The receiver is located $200 \mathrm{~m}$ below the right fracture tip. The source is a $100 \mathrm{~Hz}$ Ricker wavelet oriented in the $-z$ direction. The fracture is $320 \mathrm{~m}$ long with normal and shear compliances equal to $7.16 \times 10^{-10} \mathrm{~m} / \mathrm{Pa}$.

The effects of a single, vertical, finite length fracture on an incident P-wave wave were examined using the finite difference method. The incident $\mathrm{P}$-wave is generated by a 100 $\mathrm{Hz}$ explosion source. The fracture is $54.4 \mathrm{~m}$ in length and was assigned properties representative of an open, fluid-filled fracture (normal compliance $Z_{N} \square 0$ and shear fracture compliance $Z_{T} \rightarrow \infty$ ). The velocities in the model are $3000 \mathrm{~m} / \mathrm{s}$ and $1731 \mathrm{~m} / \mathrm{s}$ for the $P$ - and $S$-waves, respectively, and the density is $2100 \mathrm{~kg} / \mathrm{m}^{3}$.

Figure 4 displays the vertical component of the particle velocity for three source locations. When the source is located off the fracture plane (Figure $4 \mathrm{~b}$ ), the incident Pwave generates converted P-S reflected and P-S transmitted waves. The magnitude of these waves appears much larger than the fracture tip diffracted waves. When the source is located directly above the fracture, the P-S converted waves are no longer excited (Figure 4a). For this case of grazing incidence, we have found that it is possible to excite a P-S fracture head wave, but only when the fracture has a non-zero normal compliance (i.e., $Z_{N}>0$ ), as might be the case for a gas-filled fracture (case not shown). 


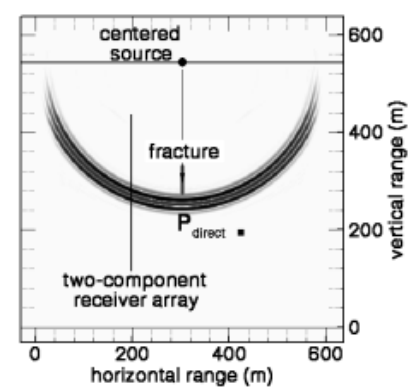

(a)

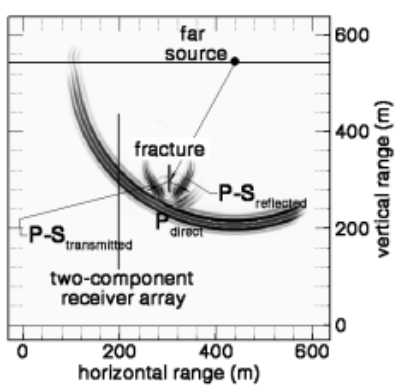

(b)

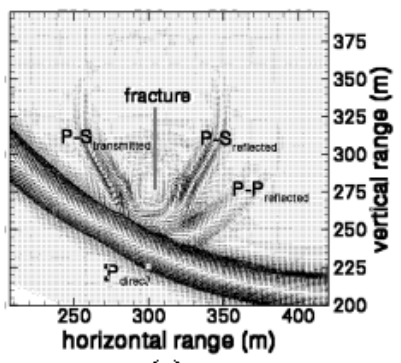

(c)

Figure 4. Snapshots of the vertical particle velocity produced by a $100 \mathrm{~Hz}$ explosion source for three source-receiver offsets: (a) fracture-centered source, and (b) offset source (angle of incidence is $62^{\circ}$ ). The fracture is $54.4 \mathrm{~m}$ in length with normal and shear fracture compliances $Z_{N} \square 0$ and $Z_{T} \rightarrow \infty$, representative of an open, fluid-filled fracture. An expanded view of (b) is shown as a vector plot in (c). The magnitudes of the fractureconverted waves are near-equal, as predicted by plane wave theory (Figure 5b).

An expanded view of the wavefield of Figure $4 \mathrm{~b}$ is displayed as a vector plot of the particle velocities in Figure 4c. Faint evidence of fracture tip diffractions and fracture interface waves are present, with stronger P-S converted waves and a P-P reflection. The basic character of the wavefield suggests that the dominant seismic signatures for this particular problem geometry and fracture properties are the converted P-S waves. For this particular model, the amplitudes of the transmitted and reflected P-S waves and the reflected P-P wave have similar magnitudes, as predicted by plane wave theory (Figure $5 b)$.

\subsection{Seismic Visibility of Discrete Fractures}

The finite difference fracture modeling described in the previous section indicates that a dominant seismic signature of a discrete fracture is the P-S converted waves. Fracture tip diffracted waves, while generated, were observed to be weak and, additionally, to suffer geometric spreading similar to waves emerging from a point source. Similar modeling results demonstrate that fracture interface waves (Pyrak-Nolte et al., 1992) can be generated when the source is located near the fracture, and via body wave conversions at the fracture tips. Fracture interface waves are localized elastic disturbances that propagate along the fracture with amplitudes that decay rapidly away from the fracture. These waves radiate waves off the fracture in the form of tip diffractions, which are again weak in comparison to the P-S converted waves. Because the P-S converted waves appear to be a dominant signature of discrete fractures, we will focus our efforts on using 
these waves to image discrete fractures.

The principal geometric factors controlling the magnitude of converted waves generated by discrete, finite-length fractures are: the fracture length $l$, and the equivalent background thickness of the fracture $\ell$ (Haugen and Schoenberg, 2000). 2-D numerical simulations of wave propagation in a medium containing finite length fractures indicate that the fracture length should be greater than approximately one half of the P-wave wavelength to generate appreciable P-S converted waves, i.e., a dimensionless wavenumber of magnitude $k_{P} l>3$, where $k_{P}$ is the $\mathrm{P}$-wave wavenumber and $l$ is the length of the fracture (Figure 5a).

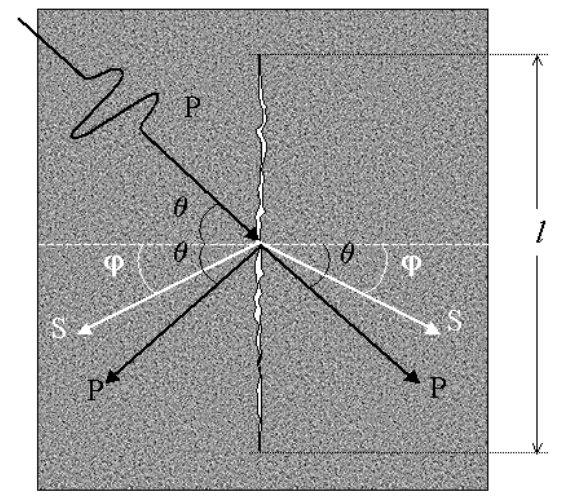

(a)

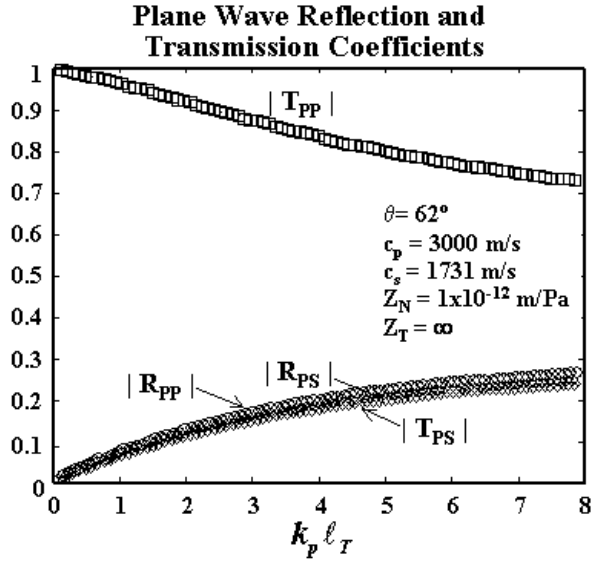

(b)

Figure 5. Plane wave transmission and reflection at a single fracture: (a) P-wave incident upon the fracture at $62^{\circ}$, and (2) transmission and reflection coefficients (assuming an infinitely long fracture) for the P-P and P-S transmitted and reflected waves computed as a function of the dimensionless wavenumber.

The influence of the fracture properties on the generation of converted S-waves by an incident $\mathrm{P}$-wave is determined by a second dimensionless wavenumber $k_{P} \ell_{T}$, where $\ell_{\mathrm{T}}=\mathrm{Z}_{\mathrm{T}} \mu$ is the tangential equivalent thickness of the fracture (Haugen and Schoenberg, 2000), $Z_{T}$ is the fracture tangential compliance, and $\mu$ is the shear modulus of the host rock. Physically, $\ell_{T}$ represents the thickness of the host medium required to achieve the same shear deformation as that taking place across the fracture. $\ell_{T}$ is a static property of the fracture with dimensions of length that can be an order of magnitude greater than the physical width of the fracture.

Developing a general rule for the magnitude of $k_{P} \ell_{T}$ required for detectable P-S fracture converted waves is complicated by an angle dependency of the converted wave reflection and transmission coefficients that is not included in this simple dimensionless quantity. For the specific example shown in Figure 5a (fluid-filled, open fracture with a P-wave incident at $62^{\circ}$ ), the magnitude of $k_{P} \ell_{T}$ required to generate P-S converted waves can be 
estimated from the plane wave reflection and transmission coefficients for a single, infinitely long fracture (e.g., Schoenberg, 1980; Pyrak-Nolte et al., 1990; Gu et al., 1996b), as shown in Figure 5b. Examination of the P-S reflection and transmission coefficients in Figure $5 \mathrm{~b}$ shows that P-S converted waves with amplitudes approximately $10 \%$ to $30 \%$ of the incident $\mathrm{P}$-wave are produced when $k_{P} \ell_{T}>1$.

\subsection{Numerical Results for a Vertically Fractured Gas Reservoir with Passive Seismic Illumination}

To examine the effects of multiple, finite-length fractures on an incident body wave, we performed a series of numerical simulations on simple layered media containing a single set of vertical fractures. In these simulations, layers containing vertical fractures were illuminated from below by a vertically incident P-wave. Near vertical incidence is expected even for seismic waves generated by local earthquakes located less than $100 \mathrm{~km}$ away because the strong velocity gradient in the upper $10 \mathrm{~km}$ which serves as a lens that turns waves to near vertical incidence.

A simple 5 layer model with a $10 \%$ variation in $\mathrm{P}$ - and S-wave velocities and containing three vertical fractures is shown in Figure 6. The average P- and S-wave velocities are $3000 \mathrm{~m} / \mathrm{s}$ and $1731 \mathrm{~m} / \mathrm{s}$, respectively, and the spatially uniform density is $2100 \mathrm{~kg} / \mathrm{m}^{3}$. The middle layer contains three fractures $54.4 \mathrm{~m}$ in height and separated by $100 \mathrm{~m}$. The fracture stiffnesses for the three fractures from left to right are: (1) $K_{N}=K_{T}=6.2 \times 10^{8}$ $\mathrm{Pa} / \mathrm{m}$, (2) $K_{N}=K_{T}=1.9 \times 10^{9} \mathrm{~Pa} / \mathrm{m}$, and (3) $K_{N}=K_{T}=4.1 \times 10^{9} \mathrm{~Pa} / \mathrm{m}$. For reference, these three values of fracture stiffness yield normal incident plane P-wave transmission coefficients equal to $0.3,0.7$, and 0.9 , respectively.

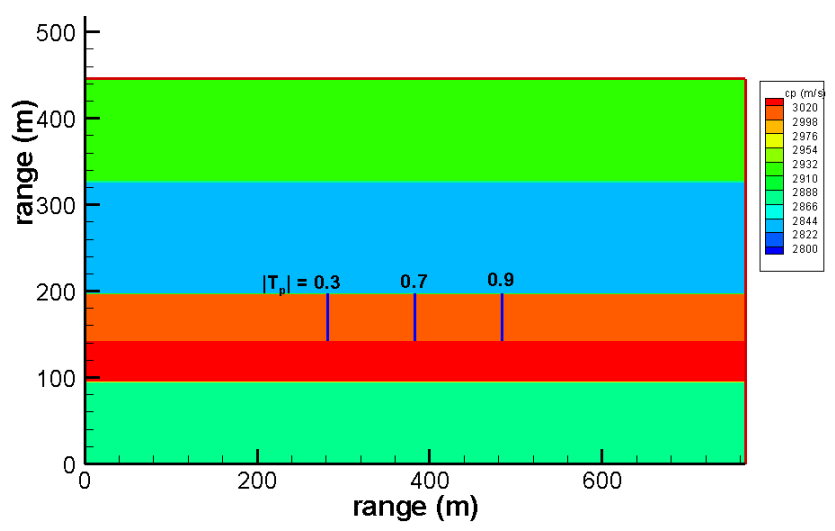

Figure 6. Layered earth model consisting of a $10 \%$ variation in velocity. The middle layer contains three fractures $54.4 \mathrm{~m}$ in height and separated by $100 \mathrm{~m}$. The fracture stiffnesses are given in the text.

Snapshots of the vertical component of the particle velocity for a $100 \mathrm{~Hz}$ plane P-wave source located below the fractured layer is displayed in Figure 7. At time step \#200, the plane $\mathrm{P}$-wave generates $\mathrm{V}$-shaped waves that are the P-S fracture converted waves and 
weaker P-P fracture tip diffracted waves. Clear separation of the direct P-wave, the P-P fracture tip diffractions, and the $\mathrm{P}-\mathrm{S}$ fracture converted waves is visible in the last snapshot (time step \#400). Although it is difficult to see in Figure 7 because of the saturated gray scale shading, the largest P-S conversions occur on the lowest stiffness fracture (left most fracture).
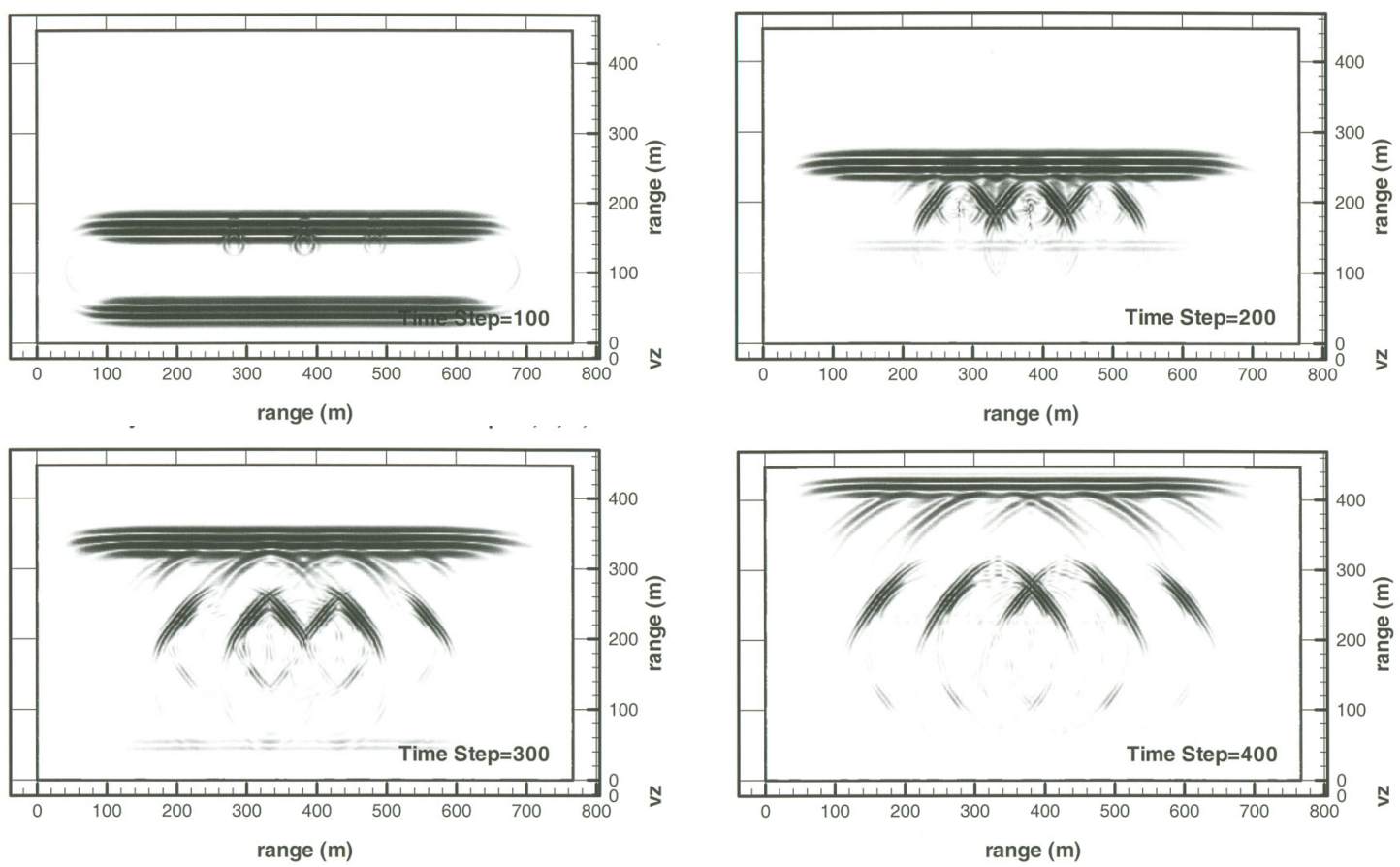

Figure 7. Snapshots of the vertical component of particle velocity generated by a plane P-wave source located below a fractured layer (Figure 6).

To further illustrate the effects of a plane P-wave normally incident upon a fractured layer, we have performed a series of simulations on simple layered media containing a single set of vertical fractures with an average fracture spacing comparable to the layer thickness (Figure 8). In these models, the fractures are randomly perturbed about a mean spacing equal to the fracture height. The fracture heights are assumed constant and equal to the bed thickness (i.e., bed-limited fractures). The fractures were assumed to have equal fracture stiffnesses (normal and shear fracture stiffnesses equal to $7.6 \times 10^{7} \mathrm{~Pa} / \mathrm{m}$ ), representative of a compliant, gas-filled fracture. The average velocities in the model are $3500 \mathrm{~m} / \mathrm{s}$ and $2143 \mathrm{~m} / \mathrm{s}$ for the $\mathrm{P}$ - and S-waves, respectively. 


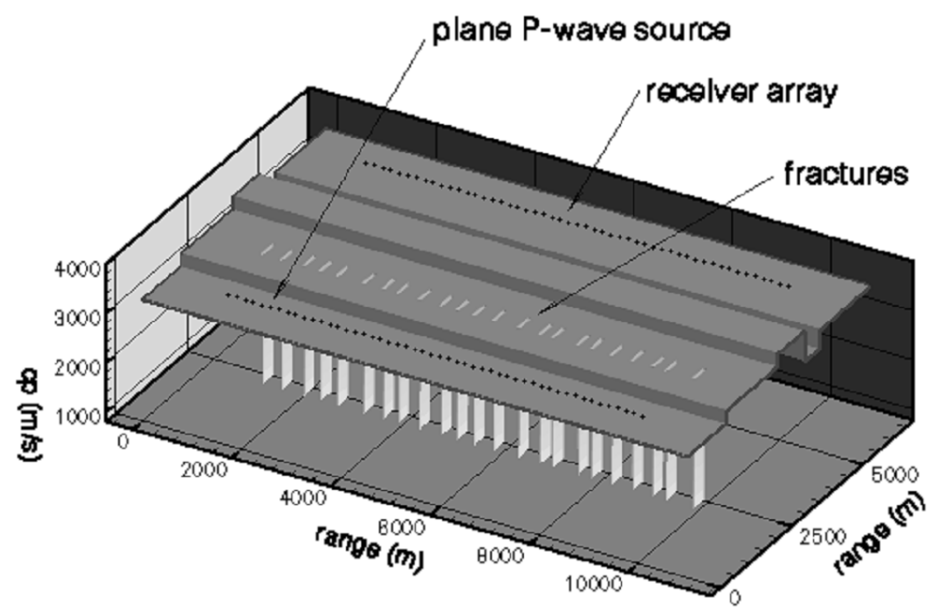

Figure 8. 2-D elastic finite difference model with vertical fractures. The source is a plane P-wave source normally incident upon the fractured layer. The receiver array is composed of 180 two-component receivers spaced $46 \mathrm{~m}$ apart. The velocity structure is comprised of 5 layers with 10\% velocity variation (average $\mathrm{P}$ - and $\mathrm{S}$-wave velocities are $3500 \mathrm{~m} / \mathrm{s}$ and $2200 \mathrm{~m} / \mathrm{s}$, respectively). Finite difference simulations for this model were performed for fracture heights of $46 \mathrm{~m}, 207 \mathrm{~m}$, and $391 \mathrm{~m}$ (shown above). Fracture spacings are stochastic with an average value equal to the fracture height. All fractures were assumed to have identical fracture stiffnesses characteristic of open, gas-filled fractures.

A snapshot of the vertical component of particle velocity is displayed in Figure 9 for a 10 $\mathrm{Hz}$, vertically-incident P-wave and a fracture height of $391 \mathrm{~m}$. The particle velocities recorded $3.3 \mathrm{~km}$ from the base of the fracture comb are shown in Figure 10 for three fracture heights: $46 \mathrm{~m}, 207 \mathrm{~m}$, and $391 \mathrm{~m}$. For a $10 \mathrm{~Hz}$ wave, these fracture heights correspond approximately to $1 / 4 \lambda_{s}, 1 \lambda_{s}$, and $2 \lambda_{s}$, respectively, where $\lambda_{s}$ is the wavelength of the shear wave. The P-to-P and P-to-S scattered waves can be clearly seen in these waveforms for the $207 \mathrm{~m}$ and $391 \mathrm{~m}$ fracture height cases, but are very weak for the $46 \mathrm{~m}$ case. From these simulations, it can be concluded that the P-to-P and P-to-S scattered waves are discernable when the thickness of the fractured layer is on the order or larger than the shear wave wavelength, and become small as the layer thickness drops to onefourth of the shear wave wavelength. Thus, for typical reservoir thicknesses of 10 to 50 $\mathrm{m}$ and an assumed average shear wave velocity of $2000 \mathrm{~m} / \mathrm{s}$, the required bandwidths of the passive waves for appreciable fracture-generated scattered waves are on the order of 40 to $200 \mathrm{~Hz}$. These frequencies are probably above the upper limit of what is detectable by a surface array at field site with typical near-surface attenuation. Based on these results, the prospects for observing fracture-generated scattered waves on a multicomponent surface sensor array with $10 \mathrm{~Hz}$ waves is feasible for large scale discontinuities, such as faults and massive fractures that have vertical extents on the order of several hundreds of meters. 


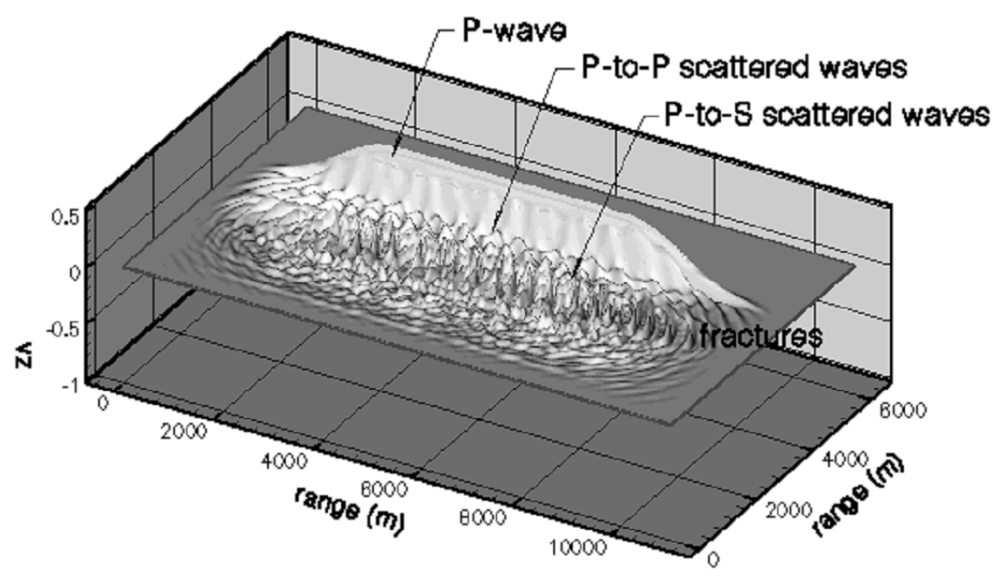

Figure 9. Snapshot of the vertical component of the particle velocity at $0.9 \mathrm{~s}$ for the fracture model shown in Figure 1 (fracture height=391 m). Note the fracture-generated P-to-P and P-to-S scattered waves following the direct P-wave.
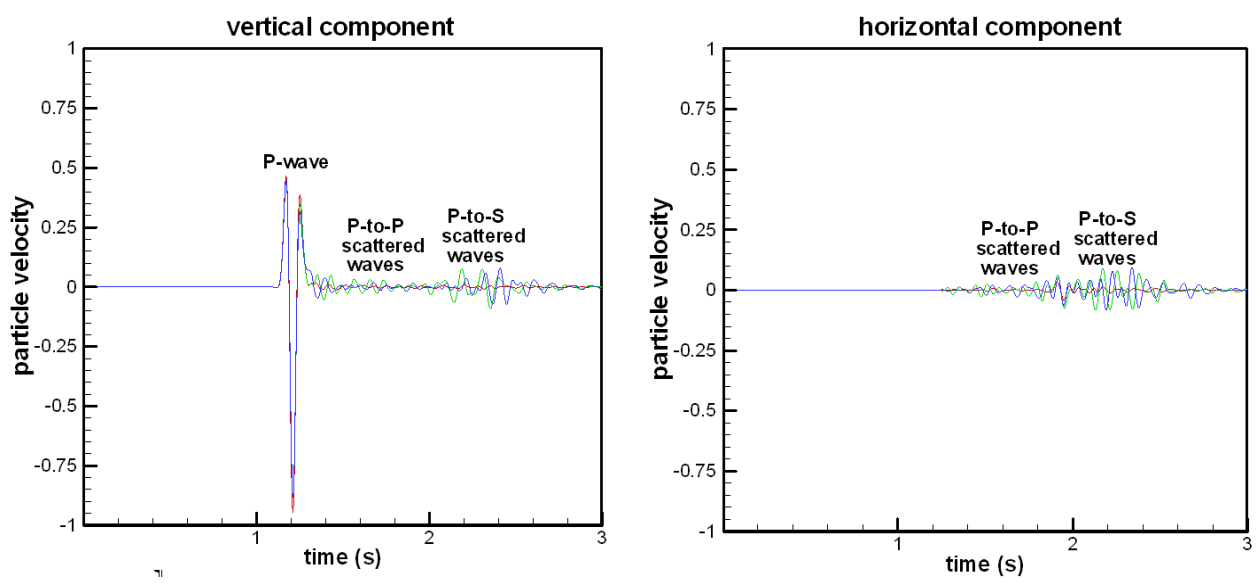

Figure 10. Particle velocities recorded by a receiver in the center of the array at a distance of $3.3 \mathrm{~km}$ from the base of the fracture comb. The amplitudes of the P-to-P and P-to-S scattered waves become small when the height of the fracture comb is reduced from approximately $2 \lambda_{\mathrm{s}}$ to $\lambda_{\mathrm{s}} / 4: 391 \mathrm{~m}$ (blue), $207 \mathrm{~m}$ (green), and $46 \mathrm{~m}$ (red).

\section{Passive Seismic Array Design Considerations}

If we accept that massive, compliant fractures with heights on the order of several hundred meters exist in the subsurface and that 10 to $20 \mathrm{~Hz}$ waves are sufficient for their generation of scattered waves, we can determine the characteristics of a passive seismic array that can be used to image these structures beneath the array. The Rayleigh criterion gives a conservative estimate of the separation at which two point sources with a spacing $\mathrm{d}$ can be distinguished from one another: $d \approx \lambda Z / L$, where $\lambda$ is the wavelength, $Z$ is the distance from the array to the point scatterers, and $L$ is the aperture of the array (Figure 11). The wavelength of a $20 \mathrm{~Hz} \mathrm{~S}$-wave, assuming an average velocity of $2000 \mathrm{~m} / \mathrm{s}$, is 
$100 \mathrm{~m}$. If we desire to resolve structures with a minimum spacing of $200 \mathrm{~m}$ (i.e., $d=200$ $\mathrm{m}$ ), and, in addition, we want to see these structures at a depth of $2 \mathrm{~km}$ (i.e., $Z=2000 \mathrm{~m}$ ), then the size of the array required for this purpose, according to the Rayleigh criterion, is $L=\lambda Z / d=1000 \mathrm{~m}$. Assuming receivers with a one-half shear wavelength spacing $(50 \mathrm{~m})$, a 2-D passive array with receivers spaced on a regular grid would require on the order of 400 receivers.

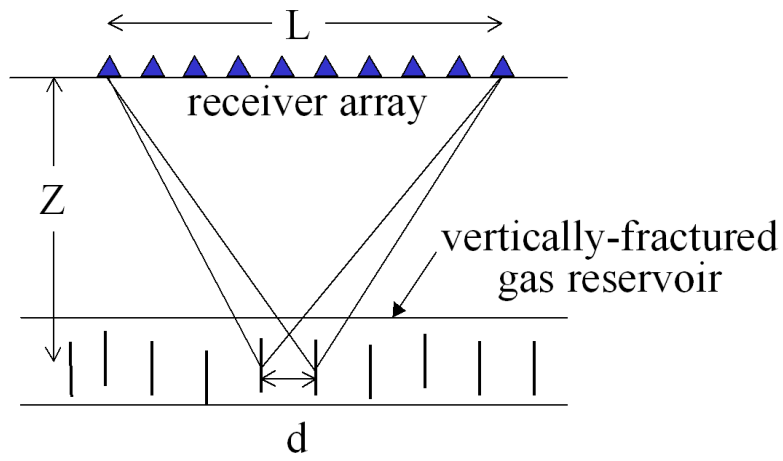

Figure 11. The transverse spatial resolution of the array to first order is proportional to the depth of the fractured layer $Z$ and the wavelength $\lambda$, and inversely proportional to the aperture of the array $L$.

At present, the cost to deploy and operate such a large array of multi-component sensors is probably not competitive with the cost of performing a higher-resolution 3-D surface reflection survey. Both technologies utilize similar sensors and data acquisition systems, and the extra cost of running an active source is probably more than compensated for by the cost of having to leave the array in place for an extended period during which the passive monitoring is performed. Putting cost issues aside, one potential benefit of passive monitoring may be realized in regions where high attenuation precludes the use of surface seismic methods. If these regions are located near active faults, the larger energies provided by natural sources and the fact that the waves travel only once through the medium, may allow these regions to be characterized by passive seismic imaging. If, in addition, substantial reductions in the cost of multi-component sensors can be realized as the petroleum industry expands it use of permanent arrays, passive experiments may become feasible to perform on the ocean floor, where signal bandwidth can be much higher than in the near surface.

\subsection{Reverse Time Fracture Imaging with Passive P-S Converted Waves}

The objective of this subsection is to explore the possibility of imaging fractures using passive seismic data recorded by a multicomponent surface sensor array. In past studies, imaging of subsurface heterogeneities with passive seismic waves was attempted by either assuming that each point in a three-dimensional volume beneath the array is a point scatter, computing the moveout trajectory for the scattering point, and then computing the semblance over time along this trajectory in the data (Cole, 1995), or by backpropagating the recorded wavefield back into the medium using an integral representation and looking 
for focusing at each depth as the scattered waves converge on the primary wave (Troitskiy et al., 1981; Potter, 1994; Norton and Won, 2000). Both of these methods differ from conventional imaging using active sources in that they lack an imaging condition that tells the algorithm how to refocus the scattered waves at the location in the medium where they were generated.

In this section, we describe an imaging methodology that we developed for converted wave fracture imaging that is based on reverse time backpropagation of the wavefield recorded along a receiver array with multicomponent sensors. The reverse time converted wave imaging scheme for passive seismic waves does not require any knowledge of the source properties, such as location, radiation pattern, or source time function. The imaging scheme consists of the following steps:

\section{Elastic Reverse Time Fracture Imaging Scheme}

1. Separate the direct $\mathrm{P}$-wave and its coda (here we define the coda here as the part of the wavefield arriving after the direct P-wave that includes P-P and P-S waves) into two multicomponent wavefields,

2. Using a priori information about the smooth background P- and S-wave velocity structure (e.g., obtained from travel time tomography), time reverse the two multicomponent wavefields recorded along the surface sensor array such that first data in becomes the last data out,

3. Apply the two multicomponent, time-reversed array data as sources at the receiver locations in two separate elastic finite difference codes to "backpropagate" the direct P-wave and its coda,

4. Form images of heterogeneities at each grid location in the finite difference model that produce P-P and P-S scattering and conversions waves (e.g., fractures, layer boundaries) by taking the product of the backpropagated direct P-wave and coda wavefields,

5. Repeat steps 1-4 for each time step in the backpropagation of the wavefields from the receiver array into the model domain. The image of the fractures and other heterogeneities that generate P-P scattered waves and P-S converted waves is produced by summing the result of step 4 for all the time steps required to backpropagate the wavefield from the receiver array through the imaging region.

This approach for imaging fractures can be viewed as reverse time migration adapted to converted waves and transmission acquisition geometries. In standard reverse time migration scheme (Chang and McMechan, 1986, 1987), the wavefield recorded at the receiver array is first processed to mute out the direct wave, and then time-reversed and propagated back into the medium (i.e., backpropagation of the scattered wavefield). Concurrently, the wavefield from the source is forward propagated into the medium. An image of the scatterers is formed from the product of the source-forward propagated and receiver-backpropagated wavefields at each time step (a process that extracts the timeand space-coincident forward and back propagated wavefields) and adding the current 
image to the images obtained at earlier time steps. A basic limitation of this approach for passive seismic imaging is that it requires knowledge of the source location. The reverse time converted wave imaging scheme described in Steps 1-5 can be performed for transmission geometries without knowledge of the source location provided the direct Pwave and P-S converted waves are recorded by a multi-component receiver array.

As with any imaging algorithm, general knowledge of the background (low wavenumber) velocities are required (for converted wave imaging, both $\mathrm{P}$ - and S-wave velocities) to perform the backpropagation. These velocities might be obtained from traveltime tomography using the passive seismic surface array (e.g., see Aki and Richards, 1980) or by the crosscorrelation technique described by Cole (1995).

Application of this converted wave reverse time migration scheme to fracture imaging is presented in Figure 12. The model is a five layered medium with three vertical fratures with different fracture stiffnesses (Figure 12a). The model parameters are given in the figure caption. The passive source consists of a $100 \mathrm{~Hz}$ vertically incident plane P-wave from below the model. The image produced by applying Steps 1-5 is shown in Figure $12 \mathrm{~b}$. Aside from some vertical smearing, the converted wave reverse time migration scheme successfully images the three fractures. The magnitude of the converted waves produced by the fractures bears some correlation to the fracture compliances. However, because the brightest fracture (center fracture) is not the most compliant fracture, it is clear that there is an acquisition footprint that must be accounted for if the images are to be used quantitatively to infer fracture compliances.

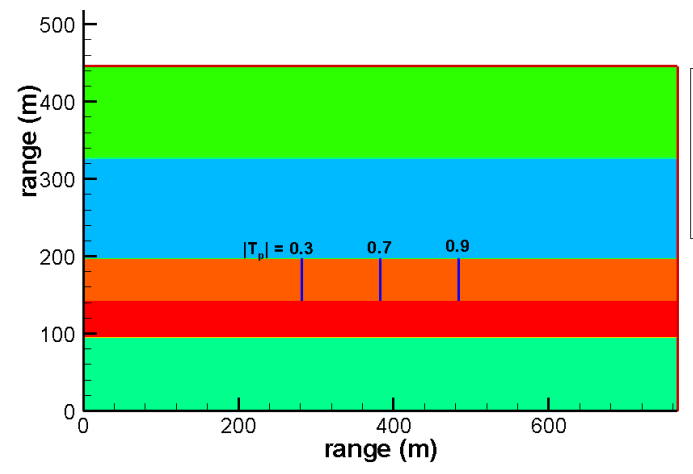

(a)

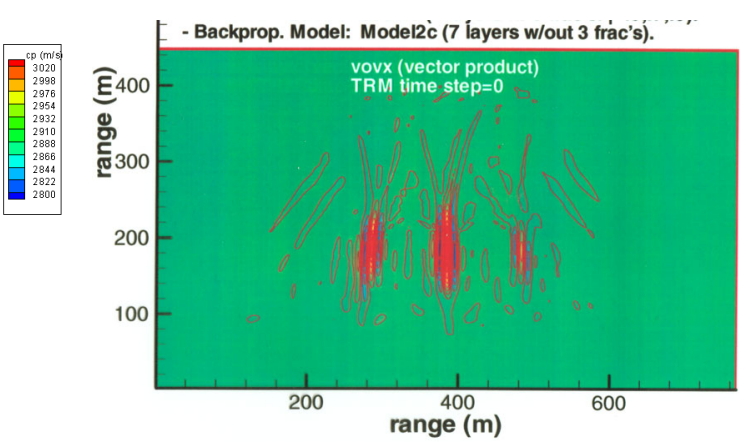

(b)

Figure 12. Passive source reverse time imaging using the seismic data recorded by a surface array for the $54.4 \mathrm{~m}\left(3 \lambda_{\mathrm{s}}\right)$ fracture height model shown in (a). The receiver array consists of 180 two-component receivers spaced $3.2 \mathrm{~m}$ apart $(576 \mathrm{~m}$ aperture). The source is a $100 \mathrm{~Hz}$, vertically incident, plane P-wave. Vertical lines in (a) show the location of the vertical fractures with their corresponding plane wave transmission coefficients for a P-wave at normal incidence. The image is formed using the reverse time imaging scheme described in steps 1-4 above. 
The second result of this passive source reverse time imaging scheme using a linear array of 180 receivers is shown in Figure 13 for the model containing the 391 m thick $\left(2 \lambda_{s}\right)$ fracture comb shown in Figure 8. The source is a $10 \mathrm{~Hz}$, vertically-incident plane Pwave incident from below. Except for some vertical smearing, the fractures are imaged using this passive source reverse time imaging algorithm. It should be noted that this approach is also applicable to active source methods provided the direct P-wave is recorded (e.g., vertical seismic profiling and crosswell seismic imaging). By using the direct wave in the imaging process, this method avoids having to estimate the characteristics of the source, such as the radiation pattern, coupling, and frequency spectrum.

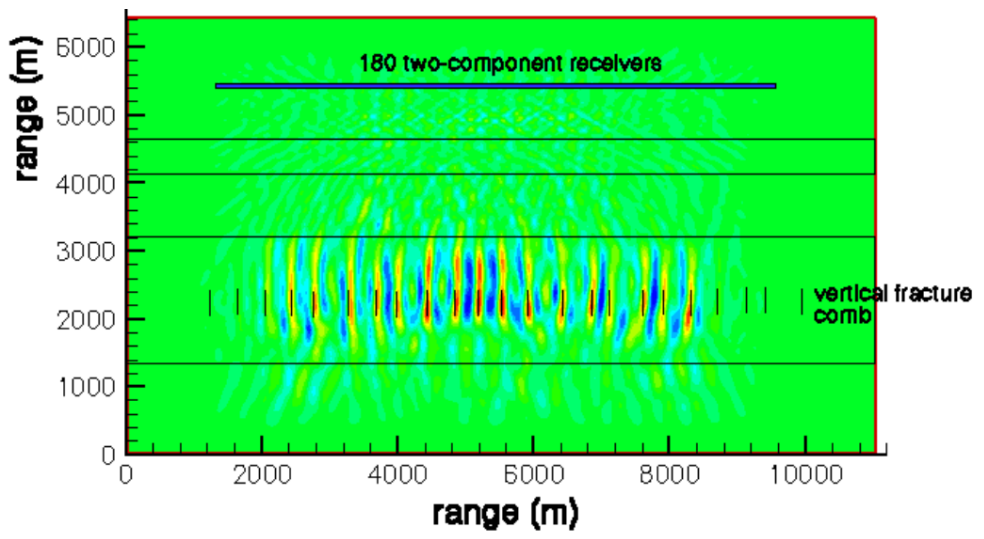

Figure 13. Passive source reverse time imaging using the seismic data recorded by a surface array for the $391 \mathrm{~m}\left(2 \lambda_{\mathrm{s}}\right)$ fracture height model shown in Figure 1. The receiver array consists of 180 two-component receivers spaced $46 \mathrm{~m}$ apart ( $8280 \mathrm{~m}$ aperture). The source is a $10 \mathrm{~Hz}$, vertically incident, plane P-wave. Horizontal lines indicate the positions of the layers shown in Figure 1. Vertical lines show the location of the vertical fractures. The image is formed by computing the vector product of the direct P-wave and the scattered P-to-P and P-to-S waves as these wavefields are backpropagated through the medium.

\section{Discussion and Conclusions}

The results of plane wave analysis and numerical (finite difference) simulations indicate that P-S converted waves are a dominant signature of discrete, vertical fractures illuminated by near-vertically incident plane waves. The amplitude of the P-S converted waves increase with fracture compliance. In this respect, gas-filled fractures will produce larger P-S converted waves than fluid-filled fractures.

The controlling factors in the application of passive seismic waves to the detection and characterization of fractured gas reservoirs are the bandwidth that can be recorded by a surface array due to near surface attenuation, and the proximity of the site to local 
seismicity. For sites where the latter factor is not an issue, the first factor places a restriction on the size of the fractures that can be resolved by the array. Fractures with dimensions on the order of the wavelength or larger, can be imaged using the time reverse imaging approach described in this article provided that the array dimensions are large enough to satisfy the transverse resolution criterion (Figure 11). For $10 \mathrm{~Hz}$ passive seismic waves and typical velocities encountered in gas reservoirs, fracture lengths should be at least several hundreds of meters. Additionally, the fracture needs to be sufficiently large to provide the required impedance contrast between the host rock and the fracture (Schoenberg, 1980). The optimum conditions are long, open, gas-filled fractures.

While a variety of methods exist for imaging subsurface structures and heterogeneities using converted waves, elastic reverse time migration is an attractive approach because it utilizes a full wave solution (i.e., finite difference method) to back propagate the wavefield. The imaging scheme presented here does not require knowledge of the source characteristics or source location.

The primary limitations of the passive seismic imaging of discrete fractures are the requirement that the prospective fractured gas reservoir be located in the proximity of areas with active seismicity, and the time required to deploy a 2-D, multicomponent surface array and record several seismic events. Given the effort and time required to deploy the multicomponent surface array, the additional cost of deployment of several active sources such as vibroseis trucks is likely to be a more cost effective option than waiting for several local seismic events to illuminate the fractured gas reservoir.

\section{Recommendations}

While there are practical limitations associated with using passive seismic events to image discrete fractures in a gas reservoirs, the basic findings of this research apply directly to the discrete fracture imaging using active sources. That is, P-S converted waves appear as dominant signatures of discrete fractures. The reverse time, elastic, converted wave imaging scheme developed in this project can be directly applied to surface, crosswell, and vertical seismic profiling geometries without significant modifications to the algorithm. The logical extension of this research is to examine the performance of this fracture imaging scheme for these source-receiver geometries.

\section{References}

Aki, K. and P.G. Richards, 1980. Quantitative Seismology, v. I - II, W.H. Freeman and Co., San Francisco.

Archuleta, R.J., S. Seale, P. Sangas, L. Baker, and S. Swain, 1992. Garner Valley downhole array of accelerometers instrumentation and preliminary data analysis, Bull. Seis. Soc. Am., 82, 1592-1621. 
Bai, T. and D.D. Pollard, 2000. Closely spaced fractures in layered rocks: Initiation mechanics and propagation kinematics, J. Struct. Geolog., 22, 1409-1425.

Chang, W.-F. and G.A. McMechan, 1986. Reverse-time migration of offset vertical seismic profiling data using the excitation-time imaging condition. Geophys., 51:67-84.

Chang, W.-F. and G.A. McMechan, 1987. Elastic reverse time migration, Geophysics, 52(10), 1365-1375.

Coates, R. T. and M. Schoenberg, 1995. Finite-difference modeling of faults and fractures, Geophysics, 60(5), 1514-1526.

Cole, S. P., 1995. Passive Seismic and Drill-Bit Experiments Using 2-D Arrays, Ph.D. Thesis, Stanford University.

Graves, R.W., 1996. Simulation seismic wave propagation in 3D elastic media using staggered-grid finite differences, Bull. Seis. Soc. Am., 86(4), 1091-1106.

Gu, B.L., Nihei, K.T., Myer, L.R. and Pyrak-Nolte, L.J. 1996a. Numerical simulation of elastic wave propagation in fractured rock with the boundary integral equation method, $J$. Geophys. Res., 101:15933-15943.

Gu, B.L., R. Suarez-Rivera, K.T. Nihei and L.R. Myer, 1996b. Incidence of plane waves upon a fracture, J. Geophys. Res., 101:25337-25346.

Haugen, G.U. and M.A. Schoenberg, 2000. The echo of a fault or fracture. Geophys. 65(1):176-189.

Kanninen, M.F. and C.H. Popelar, 1985. Advanced Fracture Mechanics, Oxford:Oxford.

Leary, P., and R. Abercrombie, 1994. Frequency dependent crustal scattering and absorption at 5-160 Hz from coda decay observed at $2.5 \mathrm{~km}$ depth, Geophys. Res. Lett., 21, 971-974.

MacBeth, C. and H.B. Lynn, 2000. Fracture-related reflectivity, Applied Seismic Anisotropy: Theory, Background, and Field Studies, Soc. Expl. Geophys., Geophysics Reprint Series No. 20:233-235.

Marfurt, K.J., R.L. Kirlin, S.L. Farmer, M.S. Bahorich, 1998. 3-D seismic attributes using a semblance based coherency algorithm, Geophysics, 63(4), 1150-1165.

Nakagawa, S., 1998. Acoustic Resonance Characteristics of Rock and Concrete Containing Fractures, Ph.D. Thesis, University of California at Berkeley.

Nihei, K.T., M. Schoenberg, W. Yi, L.R. Myer and N.G.W. Cook, 1999. Fracture channel waves, J. Geophys. Res., 104, 4769-4781.

Norton, S.J. and I.J. Won, 2000. Time exposure acoustics, IEEE Trans. Geoscience and Remote Sensing, 88(3), 1337-1343.

Pérez, M.A., V. Grechka and R.J. Michelena, 1999. Fracture detection in a carbonate reservoir using a variety of seismic methods, Geophys., 64(4), 1266-1276.

Potter, J.R., 1994. Acoustic imaging using ambient noise: Some theory and simulation results, J. Acoust. Soc. Am., 95(1), 21-33.

Price, N.J. and J.W. Cosgrove, 1990. Analysis of Geological Structures, Cambridge, 
Cambridge Univ. Press, p. 209.

Pyrak-Nolte, L.J., L.R. Myer, and N.G.W. Cook, 1990. Transmission of seismic waves across single natural fractures, J. Geophys. Res., 95, 8617-8638.

Pyrak-Nolte, L.J., J. Xu and G.M. Haley, 1992. Elastic interface waves propagating in a fracture, Phys. Rev. Lett., 68, 3650-3653.

Schoenberg, M., 1980. Elastic waves across a linear slip interface, J. Acous. Soc. Am., 68, 1516-1521.

Troitskiy, P., E.S. Husebye and A. Nikolaev, 1981. Lithospheric studies based on holographic principles, Nature, 294, 618-623.

Tsvankin, I., and H.B. Lynn, 1999. Special section on azimuthal dependence of P-wave seismic signatures-Introduction, Geophysics, 64(4), 1139-1142.

Zhang, Ch. and J.D. Achenbach, 1988. Scattering by multiple crack configurations, J. Appl. Mech., 55, 104-110. 